\title{
Polifenoles totales de Ganoderma lucidum cultivado en residuos de Pandala talaumanariñensis y Avena sativa*
}

\section{Total polyphenols of Ganoderma lucidum cultivated in wastes of Pandala talaumanariñensis and Avena sativa}

MUESES-MAFLA, ROBINSON-BYRON'; BENAVIDES-CALVACHE, OLGA-LUCIA²

Historial del artículo

Recibido para evaluación: 4 Abril 2020.

Aprobado para publicación: 12 Noviembre 2021.

* Título Proyecto de origen: "Determinación de principios activos medicinales del hongo Ganoderma lucidum cultivado sobre residuos sólidos y su uso en la producción de un alimento funcional”. Financiación: Vicerrectoría de Investigaciones VIPRI Universidad de Nariño (Colombia). Culminación: 2020

1 Universidad de Nariño, Facultad de Ingeniería Agroindustrial, Grupo de Investigación en Biotecnología Agroindustrial y Ambiental (Biota). Ingeniero Agroindustrial. Pasto, Colombia. https://orcid.org/0000-0002-3036-7191

2 Universidad de Nariño, Facultad de Ingeniería Agroindustrial, Directora Grupo de Investigación en Biotecnología Agroindustrial y Ambiental (Biota). Ingeniera Química, M.Sc.Químicas, M.Sc.Agrarias. Pasto, Colombia. https://orcid.org/0000-0002-6327-5832

Correspondencia: byronmueses14@udenar.edu.co,olgalucia@udenar.edu.co

Cómo citar este artículo: MUESES-MAFLA, ROBINSON-BYRON; BENAVIDES-CALVACHE, OLGA-LUCIA. Polifenoles totales de Ganoderma lucidum cultivado en residuos de Pandala talaumanariñensis y Avena sativa. Revista Biotecnología en el Sector Agropecuario y Agroindustrial, v. 20, n. 1, 2022, p. 18-26. Doi: https://doi.org/10.18684/rbsaa.v20.n1.2022.1491 


\section{RESUMEN}

Ganoderma lucidum es un hongo medicinal con gran demanda en el mercado mundial, reconocido por poseer numerosos compuestos bioactivos con potencial antioxidante, principalmente por sus polifenoles, triterpenos y polisacáridos. En el presente estudio se determinó el contenido de polifenoles totales mediante el método de Folin-Ciocalteu y la evaluación de grupos funcionales estructurales mediante espectroscopía infrarroja por transformada de Fourier (FTIR) en extractos etanólicos y acuosos del cuerpo fructífero de G. lucidum obtenidos del cultivo en sustrato lignocelulósico de aserrín de Pandala talaumanariñensis y paja de Avena sativa. El contenido de polifenoles totales fue mayor en los extractos acuosos (32,44 \pm 0,7023 mg EAG/g) lo que indicaría que otros compuestos bioactivos de G. lucidum con actividad antioxidante podrían ser arrastrados en el agua caliente, enriqueciendo el extracto. Los espectros IR de G. lucidum presentaron bandas de absorción en el rango de 3372,59 a 670,27 cm-1, las cuales resultaron comparables con información espectral IR de productos comerciales del género Ganoderma. Los resultados encontrados muestran un interesante potencial de G. lucidum como fuente de antioxidantes naturales que pueden usarse en la industria alimentaria y medicinal, dado su contenido de polifenoles totales.

\section{ABSTRACT}

Ganoderma lucidum is a medicinal fungus that has a worldwide demand due to its numerous bioactive components such as polyphenols, triterpenes, and polysaccharides, which have antioxidant potentials. This study assessed the total polyphenol content through the Folin-Ciocalteu method and analyzed structural functional groups using Fourier-transmform infrared spectroscopy (FTIR). Ethanolic and aqueous solutions were extracted from fruiting bodies of G. lucidum growing in lignocellulosic substrates of sawdust and straw obtained from Pandala talaumanariñensis and Avena sativa, respectively. The total polyphenol content was higher in the aqueous solutions $(32,44 \pm 0,7023 \mathrm{mg}$ $\mathrm{GAE} / \mathrm{g}$ ), which would indicate that other bioactive compounds of G. lucidum can be extracted with hot water, enriching this solution. The IR spectra of G. Iucidum showed absorption bands between the 3372,59 to $670,27 \mathrm{~cm}^{-1}$ range, which were similar to IR spectral information of the genus Ganoderma. These results demonstrate an interesting potential of G. lucidum as a source of natural antioxidants that can be used for the food and medicinal industry, given its total polyphenol content.

\section{PALABRAS CLAVE:}

Basidiomycete; Actividad antioxidante; Folin Ciocalteu; FTIR.

\section{KEYWORDS:}

Basidiomycetes; Antioxidant activity; Folin Ciocalteu; FTIR. 


\section{INTRODUCCIÓN}

Las especies de Ganoderma son hongos basidiomycetes degradantes de la madera con cuerpos fructíferos duros (Boh et al., 2007). Ganoderma lucidum también conocido como Reishi o Lingzhi es una especie de la clase Agaricomycetes, que pertenece a la familia Ganodermataceae del orden Polyphorales (Pinya et al., 2019), esta especie es rica en compuestos bioactivos con potencial medicinal, principalmente triterpenoides, polisacáridos, nucleótidos, esteroides, ácidos grasos, proteínas y glicopéptidos (Cör et al., 2018).

Las especies de Ganoderma no están clasificadas como hongos comestibles, ya que tienen un sabor amargo y dureza en sus cuerpos fructíferos y no tienen la textura carnosa característica de los hongos comestibles, pero su importante valor económico se debe a sus propiedades medicinales (Hapuarachchi et al., 2018). Actualmente, G. lucidum es utilizado como materia prima para la producción de alimentos funcionales (Uddin et al., 2019) y de las diferentes partes de su cuerpo fructífero, micelio o esporas se pueden encontrar en el mercado, diversos productos, tales como cápsulas blandas, inyecciones, tabletas, bebidas (Liu \& Zhang, 2018), polvo, té (Sangeetha et al., 2019), extractos, suplementos dietéticos (Wu et al., 2017), café, productos de esporas, jarabes, pastas de dientes, jabones y lociones, también se comercializan alimentos y suplementos farmacológicos que mejoran el sistema inmunológico y mejoran las funciones metabólicas (Hapuarachchi et al., 2018). Recientemente, los efectos farmacológicos del género Ganoderma han atraído una atención considerable por parte de la comunidad científica mundial (Zhao et al., 2019), en especial por el contenido de polisacáridos y triterpenoides de G. lucidum, que han sido reconocidos como una fuente natural prometedora de compuestos inmunomoduladores, anticancerígenos y antioxidantes (Liu \& Zhang, 2019) además, numerosos autores han evidenciado que los triterpenos y polisacáridos son los principales componentes fisiológicamente activos de G. lucidum (Hu et al., 2018) y se ha demostrado que los extractos de G. lucidum en varios solventes son eliminadores de radicales libres, anti-diabéticos y antioxidantes (Sharif et al., 2017).

Los efectos de eliminación de radicales libres por G. lucidum están relacionados con su mecanismo terapéutico para prevenir y tratar enfermedades, como bronquitis crónica, hipertensión, hiperlipidemia, diabetes, hepatitis, patología tumoral y envejecimiento. Por lo tanto, la función principal de los productos de G. lucidum es inhibir las especies reactivas de oxígeno (ROS) (Lin \& Deng, 2019) mediante la actividad de varios de sus compuestos como los fenólicos (Oludemi et al., 2018). Por consiguiente, el objetivo de esta investigación fue evaluar el contenido de polifenoles totales de extractos etanólicos y acuosos del cuerpo fructífero deshidratado y molido de Ganoderma lucidum que fue conservado en refrigeración por un periodo de seis años, así como la determinación de grupos funcionales estructurales mediante espectroscopía infrarroja por transformada de Fourier (FTIR); lo anterior para la evaluación de la estabilidad química de bioactivos presentes en G. lucidum.

\section{MÉTODO}

\section{Localización}

El trabajo se realizó en el laboratorio e invernadero del Grupo de Investigación en Biotecnología Agroindustrial y Ambiental (BIOTA) de la Universidad de Nariño en la ciudad de San Juan de Pasto, Colombia, ubicada a una altura de 2.527 m.s.n.m, con una temperatura promedio de $12^{\circ} \mathrm{C}$, y precipitación anual de $1.059 \mathrm{~mm}$ (IDEAM Pasto). 


\section{Material fúngico}

Se emplearon basidiomas de Ganoderma lucidum (cepa WC806 UPenn) deshidratados, molidos y conservados en refrigeración, obtenidos del cultivo en el invernadero del Grupo BIOTA (año 2014), en sustrato lignocelulósico con la siguiente composición en base seca: 78 \% aserrín de Pandala talaumanariñensis, 20 \% paja de Avena sativa, $1 \%$ de sacarosa y $1 \%$ de $\mathrm{CaCO}_{3}$, siendo la humedad de los sustratos de cultivo del $70 \%$.

\section{Preparación de los extractos}

Los extractos se prepararon de acuerdo a la metodología de Santillán et al. (2017). Para la obtención de los extractos etanólicos (EE) se adicionaron $2 \mathrm{~g}$ de muestra seca del basidioma en polvo, en $100 \mathrm{~mL}$ de etanol al $90 \%$ durante $24 \mathrm{~h}$ con agitación magnética en oscuridad y temperatura ambiente, se filtró y aforó a $100 \mathrm{~mL}$ con etanol al $90 \%$. Los extractos acuosos (EA) se prepararon agregando $2 \mathrm{~g}$ de muestra seca del basidioma en polvo, en $100 \mathrm{~mL}$ de agua destilada en ebullición, de igual forma se filtró y aforó a $100 \mathrm{~mL}$ con agua destilada. Se prepararon tres muestras por cada extracto que fueron simbolizadas como $\mathrm{EE}_{n}(n=1,2 \circ 3)$ y $\mathrm{EA}_{n}(n=1,2 \circ 3)$, las cuales cada una fueron evaluadas por triplicado.

\section{Contenido de polifenoles totales}

La cuantificación de polifenoles totales se realizó por el método de Folin Ciocalteu (F-C), utilizando $\mathrm{Na}_{2} \mathrm{CO}_{3}$ al 20 \% p/v como buffer, la curva de calibración se elaboró utilizando una solución de ácido gálico a 1000 ppm y, a partir de esta solución, se prepararon soluciones de 50,100, 300, 500 y 700 ppm de ácido gálico (GA) en agua destilada tipo II. En un matraz se agregó $1 \mathrm{~mL}$ de muestra, $1 \mathrm{~mL}$ de reactivo de Folin-Ciocalteu, $4 \mathrm{~mL}$ de solución de $\mathrm{Na}_{2} \mathrm{CO}_{3}$ y se aforó a $10 \mathrm{~mL}$ con agua destilada, se calentó a $50^{\circ} \mathrm{C}$ en baño María por 10 min y se midió la absorbancia a $765 \mathrm{~nm}$ en un espectrofotómetro marca Thermo scientific genesys 10 UV (Santillán et al., 2017). Las muestras se analizaron de igual forma sustituyendo el patrón GA por $1 \mathrm{~mL}$ del extracto a evaluar. El resultado promedio de las lecturas se expresó en mg equivalentes de ácido gálico por gramo de muestra (mg GAE/g).

\section{Espectros FTIR}

G. lucidum en polvo fue mezclado uniformemente con cristal $\mathrm{KBr}$, la mezcla se presionó manualmente hasta formar la tableta y la medición se realizó en un espectrofotómetro IR Affinity 1S. El espectro FTIR es generalmente aceptable cuando se logra una transmisión del 60 al 70 \% (Choong et al., 2013).

\section{Análisis estadístico}

Los datos se analizaron con el software Statgraphics ${ }^{\circledR}$ Centurion XVI. Los valores de polifenoles totales para los extractos se evaluaron con el ANOVA unidireccional y el de Tukey. Los valores de P inferiores a 0,05 se consideraron estadísticamente significativos.

\section{RESULTADOS}

Al realizar la evaluación del contenido de polifenoles totales, según el ANOVA unidireccional, no se encontraron diferencias estadísticamente significativas $(p=0,1217)$ entre la media del contenido de polifenoles totales ( $\mathrm{mg}$ GAE/g) y el tipo de extracto, con un nivel del 95,0 \% de confianza. En la figura 1 se observa que no existen dichas diferencias, no obstante, la concentración de polifenoles totales de G. lucidum en los extractos acuosos (EA) fue más alta frente a los extractos etanólicos (EE), tal como se evidencia en el Cuadro 1. 


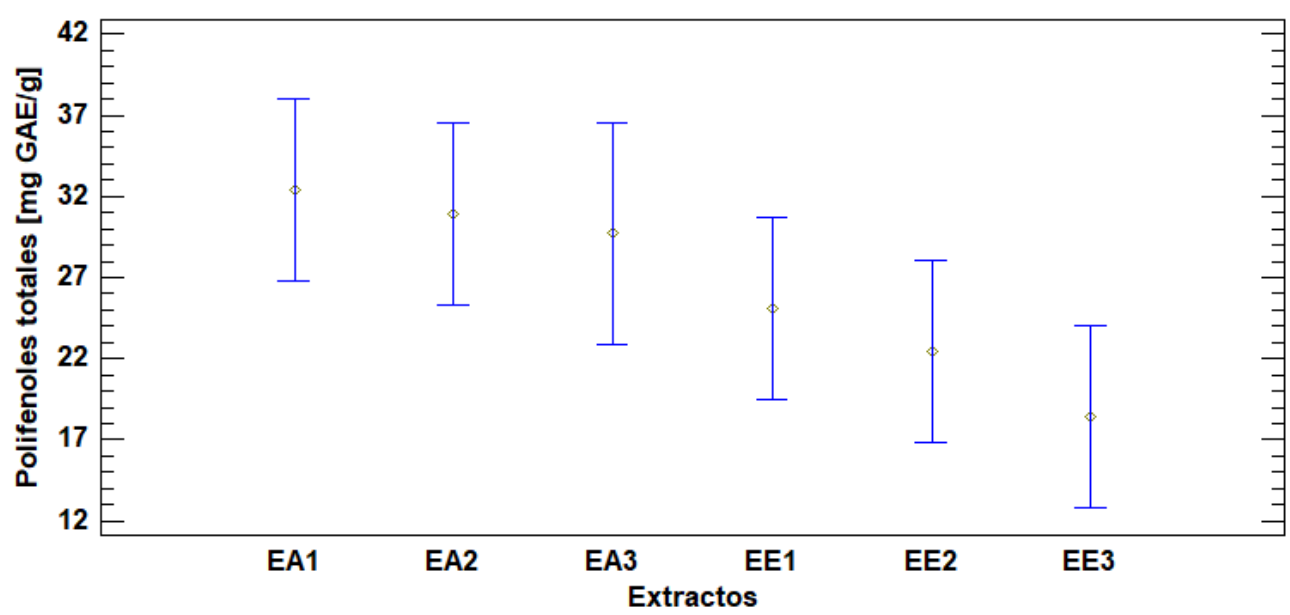

Figura 1. Gráfico de medias de polifenoles totales expresado en miligramos equivalentes de ácido gálico por gramo de muestra.

Cuadro 1. Valores promedio de polifenoles totales según el tipo de extracto

\begin{tabular}{|l|l|l|}
\hline \multicolumn{1}{|c|}{ No. de Muestra } & \multicolumn{1}{c|}{$\begin{array}{c}\text { EA } \\
\text { (mg GAE/g) }\end{array}$} & $\begin{array}{r}\text { EE } \\
\text { (mg GAE/g) }\end{array}$ \\
\hline 1 & $32,44 \pm 0,70$ & $25,11 \pm 9,24$ \\
\hline 2 & $30,96 \pm 3,38$ & $22,44 \pm 8,17$ \\
\hline 3 & $29,71 \pm 2,12$ & $18,39 \pm 6,90$ \\
\hline
\end{tabular}

Los resultados encontrados en este estudio para los extractos acuosos fueron superiores a los de otras investigaciones. Rašeta et al. (2016) determinaron el contenido de polifenoles totales en extractos acuosos de cuerpos fructíferos de G. applanatum de 21,07 $\pm 0,42 \mathrm{mg} \mathrm{GAE} / \mathrm{g}$ y $11,38 \pm 0,67 \mathrm{mg}$ GAE/g para G. lucidum. En cuanto a extractos alcohólicos de hongos del género Ganoderma, se reportan valores mayores a los de la presente investigación. Mishra et al. (2014) reportaron un valor de 28,27 mg GAE/g en peso seco en extractos metanólicos de $G$. lucidum, mientras que Huerta et al. (2016) reportaron 49,15 $\pm 0.17 \mathrm{mg} \mathrm{GAE} / \mathrm{g}$ en extractos etanólicos de G. curtisii.

El tipo de solvente tiene una influencia significativa en la dinámica del proceso de extracción y la composición química del extracto (Veljović et al., 2019). Pese a no encontrar diferencias estadísticamente significativas según el tipo de solvente empleado en este estudio, se evidenció una mayor concentración de polifenoles totales en los extractos con agua caliente, contrariamente a que los polifenoles son más solubles en disolventes orgánicos polares como el etanol y metanol (Sudheer et al., 2019). Esto apunta a que en los extractos acuosos obtenidos se pudieron arrastrar otros compuestos como los polisacáridos, comúnmente asociados a los polifenoles, ya que los macrohongos poseen triterpenos y polisacáridos en sus estructuras especializadas para la formación de esporas (Santillán et al., 2017), siendo estos últimos altamente solubles en agua caliente (Veljović et al., 2019).

La actividad antioxidante en basidiomicetes se ha relacionado con la presencia de compuestos de bajo peso molecular (polifenoles), pero también se atribuye al contenido de triterpenos y polisacáridos de alto peso molecular (Santillán et al., 2017). Lo anterior indica que, tanto el disolvente utilizado como la temperatura, influyen en la extracción de polifenoles como de otros compuestos del basidioma de G. lucidum y de esta forma la actividad antioxidante en extractos acuosos del hongo, puede ser mayor en comparación con su valor en extractos puros. 
Lee y Lim (2019) encontraron valores más altos en el contenido de polifenoles totales en extractos acuosos de G. Iucidum (33,1 $\pm 1,9 \mathrm{mg}$ GAE/g) que en el extracto de polisacáridos de G. lucidum (5,8 \pm 0,6 mg GAE/g), dada la pérdida de compuestos activos como consecuencia del proceso de purificación.

Por otra parte, el aumento del contenido de polifenoles totales en el extracto obtenido por ebullición en agua del cuerpo fructífero de G. lucidum, responde a la liberación de compuestos fenólicos, además del arrastre de compuestos insolubles, dada la descomposición celular y de enzimas oxidativas por el efecto térmico, por lo cual, para la preservación del contenido fenólico es recomendable que el proceso de extracción bajo condiciones de ebullición acuosa se realice por un tiempo inferior a cinco minutos (Minh et al., 2019).

En este estudio, los extractos acuosos del basidioma de G. lucidum se obtuvieron por ebullición durante tres minutos, reportando un valor máximo de 32,44 \pm 0,7023 miligramos Equivalente de ácido gálico por gramo de muestra en base seca ( $\mathrm{mg} \mathrm{GAE} / \mathrm{g}$ ), destacando de esta forma que el agua es un excelente solvente de extracción, no solo por disminuir costos del proceso y la contaminación derivada del uso de solventes orgánicos, sino también por la generación de extractos con elevada concentración de compuestos fenólicos. Esto, es además ventajoso en la preparación de té e infusiones de carácter alimentario, pues asegura el consumo de compuestos bioactivos con actividad antioxidante que hacen parte de G. lucidum y que son beneficiosos para la salud humana (Santa Rosa, 2016).

De hecho, los extractos acuosos en caliente de G. lucidum concentran mejor los polisacáridos y diversos estudios han concluido que estos compuestos son potentes antioxidantes (Wu \& Wang, 2009). Además, G. lucidum tiene alta actividad captadora de radicales libres, la cual está asociada a la presencia de compuestos triterpénicos que son utilizados como mecanismo de defensa contra sustancias oxidantes (Wang et al., 2019).

\section{Espectro FTIR de Ganoderma lucidum}

En el espectro infrarrojo de G. lucidum (Figura 2) se observan las siguientes bandas: $3372,59 \mathrm{~cm}^{-1} ; 2927,99 \mathrm{~cm}^{-1}$; $1651,09 \mathrm{~cm}^{-1}, 1563,33$ y también se observan varios picos seguidos entre $1500 \mathrm{~cm}^{-1}$ y $1000 \mathrm{~cm}^{-1}$.

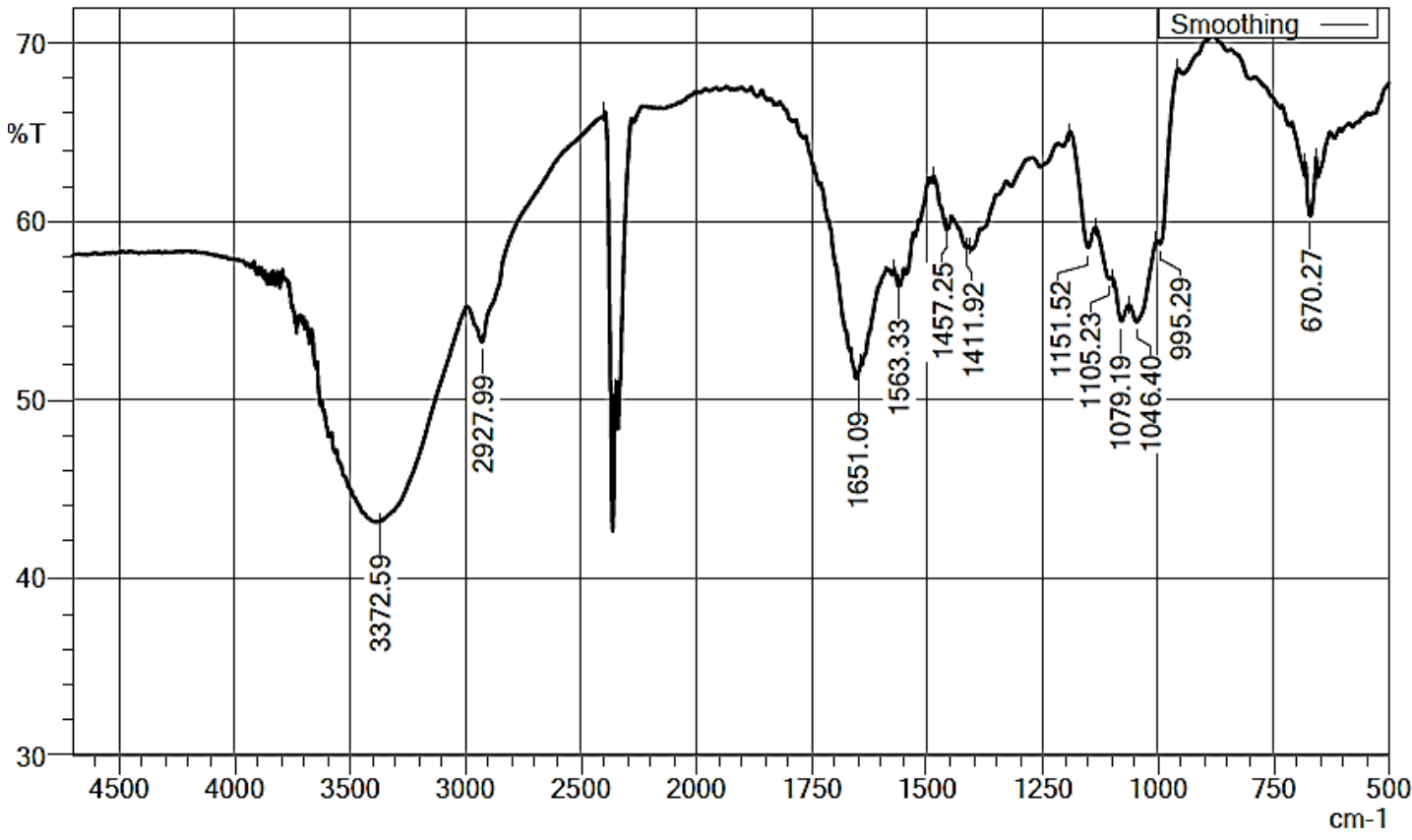

Figura 2. Espectro FTIR de Ganoderma lucidum 
El espectro FTIR de este estudio es similar al encontrado por Choong et al. (2013) a partir de un producto comercial con Lingzhi $100 \%$ natural, lo que indica que los compuestos bioactivos de las muestras empleadas en esta investigación se conservaron sin sufrir degradación durante el proceso de refrigeración del polvo de G. lucidum durante 6 años. El espectro FTIR del producto comercial con Lingzhi presentó bandas anchas y fuertes en la región de $3400-3300 \mathrm{~cm}^{-1}$ que pueden asignarse al modo de estiramiento $\mathrm{O}-\mathrm{H}$, mientras que el pico a $2925 \mathrm{~cm}^{-1}$ corresponde al estiramiento $\mathrm{C}-\mathrm{H}$. Igualmente, se ha reportado el pico a $2923 \mathrm{~cm}^{-1}$ como estiramiento de $\mathrm{C}-\mathrm{H}$ en extractos crudos de G. lucidum (Sangeetha et al., 2019). En el espectro desde $1750 \mathrm{~cm}^{-1}$ a $1500 \mathrm{~cm}^{-1}$ se encontraron picos que corresponden a amida de proteína, en el que para el producto comercial con Lingzhi se reporta un pico a $1639 \mathrm{~cm}^{-1}$ asignado a la amida I de proteínas y un pico a $1556 \mathrm{~cm}^{-1}$ asignado a la amida II (Choong et al., 2013).

En el espectro FTIR de esta investigación se observa un pico fuerte a $3372,59 \mathrm{~cm}^{-1}$ (Figura 2) que corresponde al estiramiento $\mathrm{O}-\mathrm{H} / \mathrm{C}-\mathrm{H}$ del grupo ácido carboxílico. Los grupos alquilo $(\mathrm{C}-\mathrm{H})$ y carbonilo $(\mathrm{C}=\mathrm{O})$ se observaron a $2927,99 \mathrm{~cm}^{-1}$ y $1651,09 \mathrm{~cm}^{-1}$, respectivamente y los picos en las regiones de $1780-800 \mathrm{~cm}^{-1}$ son correspondientes a amida I, amida II y proteínas.

Bandas entre 1000 y $1100 \mathrm{~cm}^{-1}$ indican la presencia de polisacáridos, más exactamente la banda $1080 \mathrm{~cm}^{-1}$, la cual se asigna al estiramiento de $\mathrm{CO}$ en $\beta$-glucanos y carbohidratos (Kozarski et al., 2012). En el presente estudio, en el espectro FTIR se observa una banda a $1079,19 \mathrm{~cm}^{-1}$, la cual indicaría la presencia de $\beta$-glucano; similar resultado fue encontrado por Ma et al., (2018) en muestras de micelios de Ganoderma liofilizadas que presentaron la banda en $1078 \mathrm{~cm}^{-1}$, relacionada al estiramiento de $\beta$-glucanos. Además, en la región de $1780-800 \mathrm{~cm}^{-1}$ se puede observar una banda fuerte en $1651,09 \mathrm{~cm}^{-1}$ que podría corresponder a la absorción de compuestos aromáticos, probablemente de los polifenoles. Kozarski et al., (2012) encontraron una banda fuerte en $1635 \mathrm{~cm}^{-1} \mathrm{en}$ compuestos aislados de $G$. lucidum, la cual indicó la presencia de compuestos fenólicos, pese a que esa región es característica de las proteínas, la banda relativamente intensa se superpuso con las vibraciones de estiramiento fenólicas.

\section{CONCLUSIONES}

Los resultados de esta investigación indican que no hay diferencia estadísticamente significativa entre el contenido de polifenoles de los extractos acuosos y etanólicos de G. lucidum, sin embargo, los extractos acuosos presentaron mayor contenido de polifenoles totales.

La concentración de polifenoles totales en extractos acuosos y etanólicos de G. lucidum, al igual que las bandas del espectro de absorción FTIR, resultaron comparables con los resultados de otras investigaciones por lo que se verifica la presencia de compuestos polifenólicos y de otros compuestos bioactivos como los polisacáridos ( $\beta$-glucanos), lo que demuestra que la refrigeración del hongo deshidratado y molido en un periodo de seis años, influye positivamente en la estabilidad química de dichos compuestos.

\section{REFERENCIAS}

BOH, M.B.; BEROVIC, J.; ZHANG.; BIN, L. Ganoderma lucidum and its pharmaceutically active compounds. Biotechnology Annual Review, v. 13, n. 7, 2007, p. 265-301. http://doi.org/10.1016/S1387-2656(07)13010-6

CÖR, D.; KNEZ, Ž.; HRNČIČ, M.K. Antitumour, antimicrobial, antioxidant and antiacetylcholinesterase effect of Ganoderma lucidum terpenoids and polysaccharides: A review. Molecules, v. 23, n. 3, 2018, p. 1-21. https://doi.org/ 10.3390/molecules23030649 
CHOONG, Y.K.; SUN, S.Q.; ZHOU, Q.; LAN, J.; LEE, H.L.; CHEN, X.D. Verification of Ganoderma (Lingzhi) commercial products by Fourier Transform infrared spectroscopy and two-dimensional IR correlation spectroscopy. Journal of Molecular Structure, v. 1069, n. 1, 2013, p. 60-72. https://doi.org/10.1016/j.molstruc.2013.11.049

HAPUARACHCHI, K.; ELKHATEEB, W.; KARUNARATHNA, S.; CHENG, C.; BANDARA, A.; KAKUMYAN, P.; HYDE, K.; DABA, G.; WEN, T. Current status of global Ganoderma cultivation, products, industry and market. Mycosphere, v. 9, n. 5, 2018, p. 1025-1052. https//doi.org/ 10.5943/mycosphere/9/5/6

HU, G.; ZHAI, M.; NIU, R.; XU, X.; LIU, Q.; JIA, J. Optimization of Culture Condition for Ganoderic Acid Production in Ganoderma lucidum Liquid Static Culture and Design of a Suitable Bioreactor. Molecules, v. 23, n. 10, 2018, e2563. https://doi.org/10.3390/molecules23102563

HUERTA, A.I.; MOLINA T.J.; GARNICA, M.G.; YAHUACA, G. Total Polyphenols and Antioxidant Activity of Ganoderma curtisii extracts. Journal of Medicinal Plants Studies, v. 4, n. 4, 2016, p. 136-141.

KOZARSKI, M.; KLAUS, A.; NIKŠIĆ, M.; VRVIĆ, M.M.; TODOROVIĆ, N.; JAKOVLJEVIĆ, D.; VAN GRIENSVEN, L.D. Antioxidative activities and chemical characterization of polysaccharide extracts from the widely used mushrooms Ganoderma applanatum, Ganoderma lucidum, Lentinus edodes and Trametes versicolor. Journal of Food Composition and Analysis, v. 26, 2012, p. 144-153. https://doi.org/10.1016/j.jfca.2012.02.004

LEE, H.R.; LIM, H. BIN. Antimutagenic and antioxidative effects of polysaccharides isolated from the water extract of Ganoderma lucidum. Journal of Applied Pharmaceutical Science, v. 9, n. 4, 2019, p.1-7. https//doi.org/10.7324/JAPS.2019.90401

LIN, Z.; DENG, A. Antioxidative and Free Radical Scavenging Activity of Ganoderma (Lingzhi). In: Lin Z., Yang B. (eds) Ganoderma and Health. Advances in Experimental Medicine and Biology, v. 1182, 2019, p. 271-297. https/doi.org/10.1007/978-981-32-9421-9_12

LIU, S.R; ZHANG, W.R. Hyperproduction of exopolysaccharides by submerged mycelial culture of Ganoderma lucidum using a solid seed grown in fine-powder of wheat bran and in vitro evaluation of the antioxidant activity of the exopolysaccharides produced. Food Science and Biotechnology, v. 27, n. 4, 2018, p. 1129-1136. https//doi.org/10.1007/s10068-018-0343-z

LIU, S.R.; ZHANG, W.R. Optimization of submerged culture conditions involving a developed fine powder solid seed for exopolysaccharide production by the medicinal mushroom Ganoderma lucidum. Food Science and Biotechnology, v. 28, n. 4, 2019, p. 1135-1145. https://doi.org/10.1007/s10068-018-0536-5

MA, Y.; HE, H.; WU, J.; WANG, C.; CHAO, K.; HUANG, Q. Assessment of Polysaccharides from Mycelia of genus Ganoderma by Mid-Infrared and Near-Infrared Spectroscopy. Scientific Reports, v. 8, n. 10, 2018, p. 1-10. https://doi.org/10.1038/s41598-017-18422-7

MINH, N.P.; NHAN, T.N.; PHA, L.P.; NGOC, N.H.; THAO, T.P. Effect of Technical Variables on the Total Phenolic and Antioxidant Activity in Cooking of Canned White Lingzhi (Ganoderma Lucidum) Fruit. Journal of Pharmaceutical Sciences and Research, v. 11, n. 3, 2019, p. 708-711.

MISHRA, K.K.; PAL, R.S.; ARUNKUMAR, R.; BHATT, C. Comparative study of antioxidant activities of cultivated and comparative study of antioxidant activities of cultivated and wild Ganoderma lucidum (W.CURT.FR.). P.karst aphyllophoromycetideae from north western indian himalayas, The Bioscan, v. 9, n. 4, 2014, p. 1601-1605.

OLUDEMI, T.; BARROS, L.; PRIETO, M.A.; HELENO, S.A.; BARREIRO, M.F.; FERREIRA, I.R. Extraction of triterpenoids and phenolic compounds from Ganoderma lucidum: optimization study using the response surface methodology. Food \& Function, v. 9, n. 1, 2018, p. 209-226. https//doi.org/10.1039/c7fo01601h

PINYA, S.; FERRIOL, P.; TEJADA, S.; SUREDA, A. Mushrooms reishi (Ganoderma lucidum), shiitake (Lentinula edodes), maitake (Grifola frondosa). In Nonvitamin and Nonmineral Nutritional Supplements, 2019, p. 517-526. https://doi.org/10.1016/B978-0-12-812491-8.00068-0 
RAŠETA, M.; KARAMAN, M.; JAKŠIĆ, M.; ŠIBUL, F.; KEBERT, M.; NOVAKOVIĆ, A.; POPOVIĆ, M. Mineral composition, antioxidant and cytotoxic biopotentials of wild-growing Ganoderma species (Serbia): $G$. lucidum (Curtis) P. Karst vs. G. applanatum (Pers.) Pat. International Journal of Food Science and Technology, v. 51, n. 12, 2016, pp. 2583-2590. https://onlinelibrary.wiley.com/doi/10.1111/ijfs.13243

SANGEETHA, B.; KRISHNAMOORTHY, A.S.; AMIRTHAM, D.; SUNDARA-SHARMILA, D.J.; RENUKADEVI, P.; MALATHI, V. FT-IR Spectroscopic Characteristics of Ganoderma lucidum Secondary Metabolites. Journal of Applied Science and Technology, v. 38, n. 6, 2019, p. 1-8. https//doi.org/10.9734/cjast/2019/v38i630453

SANTA ROSA, C. Toxicidade oral aguda do extrato aquoso de Ganoderma lucidum: avaliação pré-clínica dos efeitos neurocomportamentais, hematológicos e estresse oxidativo [Ms.c Mestre em Ciências Farmacêuticas]. BelémPA (Brasil): Universidade Federal Do Pará, programa de pós-graduação em ciências farmacêuticas, 2016, 63 p.

SANTILLÁN, M.Á.; CASTAÑEDA O.A.; ÁLVAREZ D.A.; VALENZUELA G.R.; ROMERO B.L; TORRES J.M. Estudio preliminar de la actividad antioxidante de tres especies del género Ganoderma (Polyporaceae) nativas del estado de Hidalgo, México. Scientia Fungorum, v. 46, 2017, p. 37-45. https://doi.org/10.33885/sf.2017.46.1175

SHARIF, S.; SHAHID, M.; MUSHTAQ, M.; AKRAM, S.; RASHID, A. Wild mushrooms: A potential source of nutritional and antioxidant attributes with acceptable toxicity. Preventive Nutrition and Food Science, $v$. 22, n. 2, 2017, p. 124-130. https//doi.org/10.3746/pnf.2017.22.2.124

SUDHEER, S.; ALZORQI, I.; MANICKAM, S.; ALI, A. In Mérillon JM., Ramawat K. (eds) Bioactive Molecules in Food. Bioactive Compounds of the Wonder Medicinal Mushroom "Ganoderma lucidum". Reference Series in Phytochemistry, 2019, p. 1863-1893. https://doi.org/10.1007/978-3-319-78030-6_45

UDDIN, M.; TALUKDER, R.I.; SARKAR, M.K.I.; RAHMAN, T.; PERVIN, R.; RAHMAN, M.; AKTHER, L. Effect of Solvents on Phytochemicals Content and Antioxidant Activity of Ganoderma lucidum. The Open Microbiology Journal, v. 13, n. 1, 2019, p. 10-15. https//doi.org/ 10.2174/1874285801913010010

VELJOVIĆ, S.; NIKIĆEVIĆ, N.; NIKŠIĆ, M. Medicinal Fungus Ganoderma lucidum as Raw Material for Alcohol Beverage Production. Woodhead Publishing, v. 7, 2019, p. 161-197. https://doi.org/10.1016/B978-0-12-815269-0.00006-4

WANG, C.; LIU, X.; LIAN, C.; KE, J.: LIU, J. Triterpenes and Aromatic Meroterpenoids with Antioxidant Activity and Neuroprotective Effects from Ganoderma lucidum. Molecules, v. 24, n. 23, 2019, p. 1-11. https://doi.org/10.3390/molecules24234353

WU,D.T.; DENG, Y.; CHEN, L.X.;ZHAO, J.;BZHELYANSKY, A.; LI, S.P. Evaluation on quality consistency of Ganoderma lucidum dietary supplements collected in the United States. Scientific Reports, v. 7, n. 1, 2017, p. 1-10. https//doi.org/10.1038/s41598-017-06336-3

WU, Y.; WANG, D. A New Class of Natural Glycopeptides with Sugar Moiety Dependent Antioxidant Activities Derived from Ganoderma lucidum Fruiting Bodies. Journal of Proteome Research, v. 71, 2009, p. 241-245. https//doi.org/ 10.1021/pr800554w

ZHAO, C.; ZHANG, C.; XING, Z.; AHMAD, Z.; LI, J.S.; CHANG, M.W. Pharmacological effects of natural Ganoderma and its extracts on neurological diseases: A comprehensive review. International Journal of Biological Macromolecules, v. 121, 2019, p. 1160-1178. https://doi.org/10.1016/j.ijbiomac.2018.10.076 